\title{
Inspección autónoma de entornos submarinos
}

Recibido: 15 Febrero 2015 - Revisado: 30 Abril 2015

Aceptado: 30 Mayo 2015 - Publicado: 30 Julio 2015
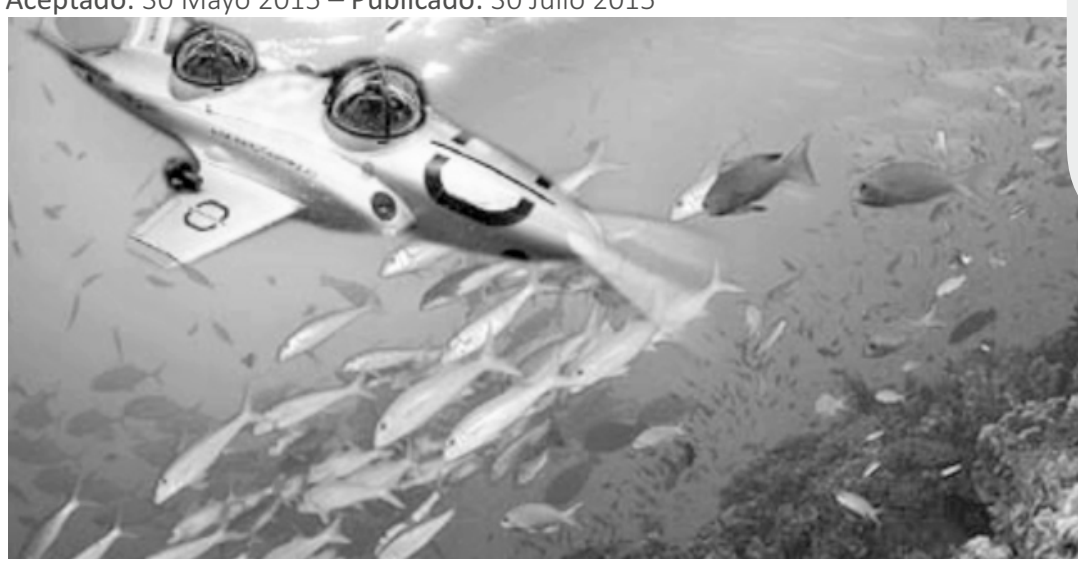

Juan David Hernández Vega

Candidato a doctor, Centro de Investigación en Visión y Robótica Submarina (CIRS), Instituto de Visión por Computador y Robótica (ViCOROB), Universidad de Girona (UdG), Carrer Pic de Peguera No 13, Código Postal: 17003, Girona, España. juandhv@eia.udg.edu

\section{Marc Carreras}

Profesor Asociado, Centro de Investigación en Vision y Robótica Submarina (CIRS), Instituto de Universidad de Girona (UdG), Carrer Pic de Péguera No 13, Código Postal: 17003, Girona, España. marc.carreras@udg.edu

Resumen: Este trabajo presenta una estrategia para la inspección de entornos marinos mediante el uso de vehículos submarinos autónomos (Autonomous Underwater Vehicle, AUV). Los entornos a explorar se asumen como desconocidos, lo que requiere que el vehículo tenga la capacidad de mapear y planificar caminos libres de colisión simultáneamente y en línea. Para esto, presentamos un sistema compuesto por tres bloques funcionales de mapeo, planificación y coordinación. El primero de ellos utiliza una estructura de datos que permite representar espacios volumétricos, donde se diferencian áreas libres de obstáculos, con obstáculos y desconocidas. El segundo de los bloques funcionales utiliza un algoritmo de planificación de caminos basado en muestreos aleatorios del espacio de configuraciones (C-Space) del vehículo. El algoritmo utilizado ha sido modificado para (re)planificar los caminos de acuerdo a la información incrementalmente adquirida del entorno. Finalmente, el coordinador se encarga de iniciar los dos bloques previamente mencionados, así como enviar los puntos resultantes del planificador a los controladores del vehículo. Por último, se presenta la validación de nuestro sistema mediante resultados en entornos reales y de simulación utilizando el vehículo SPARUS-II.

Palabras clave: planificación de caminos; mapeo; cómputo en línea; entornos submarinos; inspección; AUV.

Abstract: This work presents an approach to inspect underwater environments using Autonomous Underwater Vehicles (AUVs). The environment to be explored are assumed as unknown, which requires the vehicle to be able to map and plan collision-free paths simultaneously and online. For doing so, we present a framework composed of three functional modules of mapping, path planning and coordination. The first of them uses a data structure that presents volumetric spaces and permits to differentiate free, occupied and unknown regions. The second functional module uses a sampling-based algorithm for planning collision-free paths. The algorithm has been modified for (re)planning paths according to the information incrementally gathered from the environment. The last functional module is in charged of coordinating the first two modules and also of communicating the resulting waypoints to the vehicle's controllers. Finally, simulation and real-world results using the SPARUS-II AUV are presented in order to validate our approach.

Key words: path planning; mapping; online computing; underwater environments; survey; AUV. 


\section{INTRODUCCIÓN}

Desde sus inicios, la robótica ha tenido un rol importante en diferentes áreas del desarrollo de nuestra sociedad. Los brazos manipuladores, por ejemplo, fueron desarrollados con el objetivo de realizar tareas repetitivas, de manera rápida y con un nivel de precisión superior a la alcanzada por el ser humano. Su uso, por lo tanto, fue un factor determinante en el aumento de la productividad en el sector de manufactura.

No obstante, la limitación de los robots, entendidos como brazos manipuladores, se encontraba en su restringido espacio de trabajo, determinado en gran medida por su base fija. De allí que el concepto de robot se extendiese al de sistemas capaces de percibir su entorno, procesar la información obtenida y actuar con base en dicho procesamiento (Barrientos et al., 2007). Bajo esta extensión, se inició el desarrollo de diferentes sistemas móviles (robóticos) capaces de desplazarse a lo largo de un espacio preestablecido, obteniendo y procesando, simultáneamente, información de dicho entorno. En esta nueva etapa en la robótica, se inició el desarrollo de nuevos vehículos «inteligentes» capaces de realizar misiones de manera autónoma o semiautónoma en tierra, aire y agua. Para ello, los vehículos cuentan con sistemas de navegación que les permiten desempeñar diferentes tareas en terrenos parcial o completamente desconocidos.

En lo que respecta a los entornos submarinos, actualmente existen dos tipos de robots que se utilizan de manera habitual en diferentes clases de aplicaciones. Los vehículos teleoperados o ROV (Remotely Operated Vehicles), que son controlados desde la superficie mediante un cable umbilical y, además, pueden contar con brazos manipuladores que les posibilita realizar intervenciones remotas. Su principal limitación es su alto costo de operación, debido a la necesidad de tener un buque de apoyo. Para solucionar parte de este problema, se desarrollaron los vehículos submarinos autónomos o AUV (Autonomous Underwater Vehicles), los cuales funcionan con baterías y ejecutan de manera autónoma sus misiones.

Los AUV están equipados con diferentes sensores para realizar mediciones de su entorno, ya sea un sonar de imágenes o de distancia, una cámara de vídeo, un CTD (Conductivity, Temperature, and Depth) o cualquier otro equipo. Además, disponen de sistemas de navegación muy precisos para estimar su posición. En la actualidad, los AUV miden la altitud mediante una sonda y modifican su profundidad para asegurarse que se encuentran dentro de los márgenes seguros. Esto permite seguir el fondo marino a pocos metros, siempre que el perfil sea suave y no escarpado. En entornos más abruptos, el robot debe navegar a mayor altitud y por lo tanto, no es posible tomar imágenes visuales, debido a la reducida visibilidad del agua. Solamente los sensores acústicos, los cuales tienen menor resolución y nitidez, podrían utilizarse en estos casos.

En resumen, un AUV no puede inspeccionar estructuras o entornos naturales submarinos ( $p$. ej. arrecifes de coral, cavernas o rocas submarinas) a corta distancia, especialmente cuando no se cuenta con un mapa preciso de la zona que se desea inspeccionar. Esto representa la mayor limitación de estos vehículos en la actualidad. Además, esta tarea tampoco puede hacerse con un ROV, por los altos costos y dificultad de operar el buque de apoyo cerca de dichas estructuras.

Con base a lo anterior, este trabajo presenta una alternativa para dotar un AUV con la capacidad de inspeccionar entornos submarinos a corta distancia. Para ello, este proyecto propone el uso de una arquitectura de control, la cual incluye módulos funcionales para el mapeo y planificación de movimientos en línea, que le permiten al vehículo explorar y navegar en un entorno submarino sin previo conocimiento del mismo. 
Completada la introducción de este escrito, lo restante de este artículo se estructura de la siguiente forma. La Sección 2 presenta una revisión bibliográfica de las contribuciones más relevantes que involucran el mapeo y la planificación de caminos en vehículos submarinos. La Sección 3 explica la estrategia usada para el mapeo y planificación de caminos en línea para AUV. Allí se detalla en cada uno de los bloques funcionales involucrados, así como la forma en que éstos interactúan entre sí. Adicional a ello, en esta sección se introduce una función de optimización que permite que los caminos resultantes mantengan una distancia preestablecida respecto del entorno; de esta manera, se garantiza la visibilidad del mismo para la captura de imágenes visuales. La Sección 4 presenta y discute diferentes resultados obtenidos con el AUV SPARUS-II, un vehículo tipo torpedo (ver Fig. 1), en un escenario de simulación y del mundo real. Finalmente, la Sección 5 expone las conclusiones de este trabajo y analiza futuras extensiones del mismo.

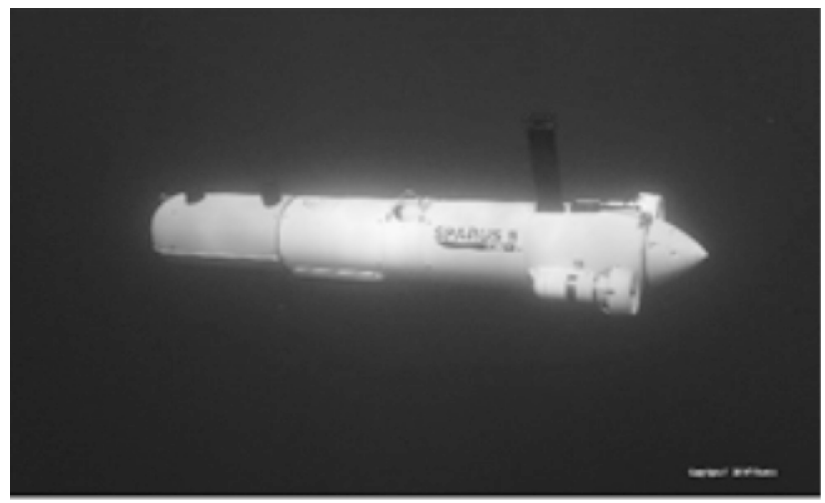

Figura 1. SPARUS-II, un AUV tipo torpedo.

\section{ANTECEDENTES}

A diferencia de lo que ocurre con los robots aéreos y terrestres, el estado actual de desarrollo de los métodos de planificación de caminos de robots submarinos es muy incipiente. Esto se debe básicamente a la dificultad en la percepción de los obstáculos que se encuentran en el medio marino. Por esta razón, en la mayoría de aplicaciones de AUV, el vehículo realiza los estudios de entornos submarinos navegando a una altitud previamente establecida, en la cual se garantiza la seguridad del mismo.

Sin embargo, el constante desarrollo de nuevas tecnologías acústicas para el mapeo 2-dimensional (2D) y 3-dimensional (3D), así como el incremento en la capacidad de cómputo disponible a bordo de los vehículos, han incentivado la propuesta de nuevas aplicaciones que requieren que los AUV operen más cerca de estructuras presentes en entornos submarinos. Entre estas nuevas aplicaciones, se destacan la inspección de estructuras como rocas submarinas (Galceran et al., 2014), cascos de barcos (Hover et al., 2012) o entornos cerrados, como cavernas (Mallios et al., 2011). No obstante, de estas aplicaciones, solo aquellas donde se pueden conocer a priori características como ubicación y forma (rocas y casco de barco) es posible realizar inspecciones de forma autónoma. Por el contrario, en casos sin información previa disponible, como las cavernas submarinas, la inspección se realiza mediante la teleoperación del vehículo.

Una alternativa para inspeccionar entornos submarinos de los cuales no se tiene información previa es realizar el mapeo y la planificación de caminos simultáneamente y en línea. Petillot et al. (2001) fueron los primeros en presentar una estrategia para la planificación de caminos y evasión de obstáculos en línea para vehículos submarinos. Para demostrar su validez, utilizaron datos reales registrados por un ROV equipado con un sonar multihaz frontal, los cuales fueron usados para calcular una trayectoria libre de colisiones y guiar un ROV en simulación. Sin embargo, la capacidad de mapeo y planificación simultánea y en línea no fue demostrada.

Maki et al. (2007) propusieron un método para la planificación de caminos en línea que utilizaba puntos característicos (o landmarks) para guiar el vehículo, 
pero en este caso, la validación experimental se hizo en un ambiente altamente controlado (tanque de agua) que carecía de características ambientales reales.

Recientemente, y con el objetivo de abordar algunas limitaciones de las contribuciones previamente mencionadas, los autores de este trabajo presentaron una estrategia para la planificación en línea de caminos libres de colisión para AUV en entornos desconocidos (Hernández et al., 2015). Allí se propuso un conjunto de tres bloques funcionales especializados, donde el primero de ellos se encarga de construir un mapa del entorno, el segundo planifica caminos utilizando dicho mapa y el tercero funciona como un coordinador entre en mapeo y la planificación.

Sin embargo, aunque esta estrategia garantiza la planificación de caminos libres de colisión, no se discute ningún criterio que demuestre que las mismas garanticen la obtención de datos útiles para una inspección submarina. Este trabajo discute una alternativa para incluir un criterio que garantice la visibilidad para la captura de imágenes de estructuras submarinas. Más detalles referentes a los bloques funcionales y este criterio de visibilidad se darán a lo largo de las siguientes secciones.

\section{METODOLOGÍA}

Esta sección resume nuestra estrategia para dotar un AUV con la capacidad de navegar en un entorno desconocido. Para lograr esto, el vehículo debe construir un mapa de su entorno, al tiempo que planifica caminos sobre éste. Adicionalmente, es necesario establecer una métrica que permite garantizar la visibilidad de una estructura submarina durante la ejecución de la trayectoria resultante.

\subsection{Estrategia para el mapeo y planificación} simultánea y en línea

La figura 2 muestra los tres bloques funcionales que constituyen nuestra estrategia de mapeo y planificación simultánea y en línea, así como la conexión entre ellos y otras partes de la arquitectura de control del vehículo.

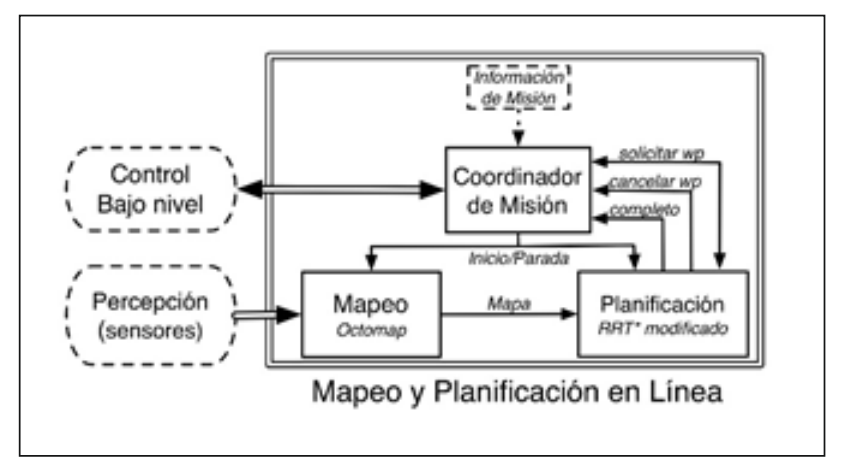

Figura 2. estrategia de mapeo y planificación simultánea y en línea para AUV compuesta por tres bloques funcionales, Coordinación, Mapeo y Planificación.

\subsubsection{Mapeo}

El bloque funcional de mapeo es el encargado de construir una representación del entorno donde el AUV ejecuta su misión. Para esto, se utiliza la información obtenida de diferentes sensores exteroceptivos del vehículo, como sonars multihaz, perfiladores, ecosondas, etc. Estos sensores proporcionan la distancia a un objeto u obstáculo detectado; así, permite determinar los espacios libres y ocupados en los alrededores del vehículo. Esta información se procesa conjuntamente con la navegación del robot (posición y orientación), para, de este modo, construir una representación 3D.

En nuestro caso, hemos decidido utilizar Octomaps para dicha representación; son estructuras de datos basados en árboles octales (octrees), que permiten modelar espacios volumétricos, diferenciando áreas 
libres, ocupadas y no exploradas (Hornung et al., 2013), lo cual resulta de utilidad en aplicaciones de planificación.

La Fig. 3 muestra el AUV SPARUS-II en un entorno de simulación junto a una serie de bloques de concreto, así como la representación (o mapa) equivalente usando Octomaps.

\subsubsection{Planificación}

La planificación de caminos es el proceso que permite calcular una serie de puntos que conectan una configuración inicial y final (o meta) del espacio de las configuraciones o C-Space (Configuration space). el CSpace se define como un espacio que contiene todas las posibles configuraciones de un robot. Por lo tanto, la planificación de caminos es el proceso que permite calcular una serie de puntos que pertenecen a regiones del C-Space que son libres de colisiones, en otras palabras, que hace parte del C-Free.

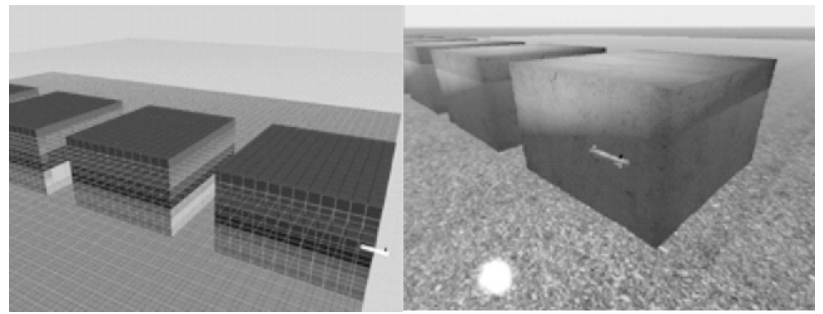

Figura 3. EI AUV SPARUS-II en un entorno de simulación.

En (a) se observa el vehículo en un entorno virtual compuesto por una serie de bloques de concreto. En (b) se aprecia la representación equivalente utilizando Octomaps (Hornung et al., 2013), donde cada uno de los vóxel tienen una resolución de $1 \mathrm{~m}$.

Para resolver esta tarea, este bloque funcional recibe una especificación del problema que incluye una posición inicial y una final, así como diferentes parámetros como tiempo de cómputo disponible, distancia mínima a la posición final, fronteras del espacio de planificación, entre otros. En nuestro caso, este módulo funcional contiene una versión modificada del algoritmo de árboles de rápida exploración aleatoria o RRT (Rapidly exploring Random Tree) (LaValle y Kuffner, 2001), pero específicamente una modificación de su variante que optimiza los caminos resultantes, RRT* (Karaman y Frazzoli, 2011).

El RRT y sus variantes, incluyendo la utilizada en este trabajo, es un algoritmo que pertenece a la familia de métodos basados en un muestreo aleatorio del CSpace. Este tipo de métodos, a diferencia de otros que calculan y verifican explícitamente y por completo el C-Space, busca agilizar dicho proceso limitando la verificación solo a las muestras aleatorias del C-Space. EI RRT está compuesto por dos procedimientos principales, muestreo y expansión. El primero de ellos genera una muestra aleatoria dentro de los límites preestablecidos del C-Space, mientras que el segundo expande el árbol de configuraciones hacia dicha configuración aleatoria por una distancia también preestablecida, de esta manera, genera una nueva configuración en el árbol (para más detalles, ver LaValle y Kuffner, 2001).

De manera similar, el RRT* cuenta con estos dos procedimientos, pero al momento de expandir el árbol, también verifica si configuraciones cercanas a la nueva configuración resultante pueden ser reconectadas para mejorar su costo asociado (para más detalles, ver Karaman y Frazzoli, 2011). La Fig. 4 muestra la expansión típica de un RRT y un RRT* en un entorno 2D, donde el C-Space del sistema es también 2D y corresponde a las coordenadas X-Y.

Los métodos de planificación de caminos basados en muestreos aleatorios del C-Space se clasifican en dos grupos principales: (a) Aquellos que buscan resolver una única consulta de inicio-a-meta (conocidos como single query), como es el caso del RRT, y (b) aquellos que pretenden resolver múltiples consultas (conocidos como multi-query), como ocurre como el mapa de rutas probabilístico o PRM (probabilistic roadmap) (Kavraki, Svestka, Latombe, y Overmars, 
1996). La principal diferencia es que aquellos destinados para resolver una única consulta generan una estructura que busca conectar una configuración inicio y una final (meta), mientras que los métodos de múltiples consultas buscan generar una estructura que cubra y describa de la mejor manera posible el CSpace.

En el contexto de la aplicación propuesta en este proyecto, el planificador debe encontrar un camino libre de colisiones que permita a un AUV, explorar un entorno submarino desconocido. La restricción más importante en esta aplicación radica en el hecho que la información del entorno es parcial y aumenta a medida que el vehículo se mueve en el entorno. Por esta razón, resulta más útil utilizar un método de una consulta que guíe el vehículo desde su posición actual, y con la información disponible, hasta una posición final preestablecida.

Por esta razón, se decidió modificar el RRT*, con el objetivo de que éste tenga la capacidad de replanificar. La nueva versión de planificación en línea del RRT* solicita periódicamente una versión actualizada del mapa del entorno y con base a ésta, verifica si alguna de las ramas del árbol de configuraciones se encuentra en colisión. Si una colisión es detectada con respecto a la nueva versión del mapa, dicha rama del árbol es descartada. Además, si alguna rama descartada contenía el camino desde la posición inicial (que corresponde al punto enviado al controlador del vehículo) hasta la meta, una nueva solución se buscará con una expansión convencional del RRT*. El proceso de descarte de ramas en colisión se puede apreciar claramente en la Fig. 5.

La capacidad de replanificar los caminos no solo se utilizan para las ramas en colisión, sino además, cuando el camino entre la posición actual del vehículo y el punto enviado al controlador no está libre de colisión, esto se explica en mayor detalle en la siguiente sección. Cabe aclarar que para que el vehículo complete la misión de exploración, el punto inicial de la consulta del planificador se irá modificando a medida que el vehículo avance hasta el momento llegar a una distancia mínima preestablecida a la meta.

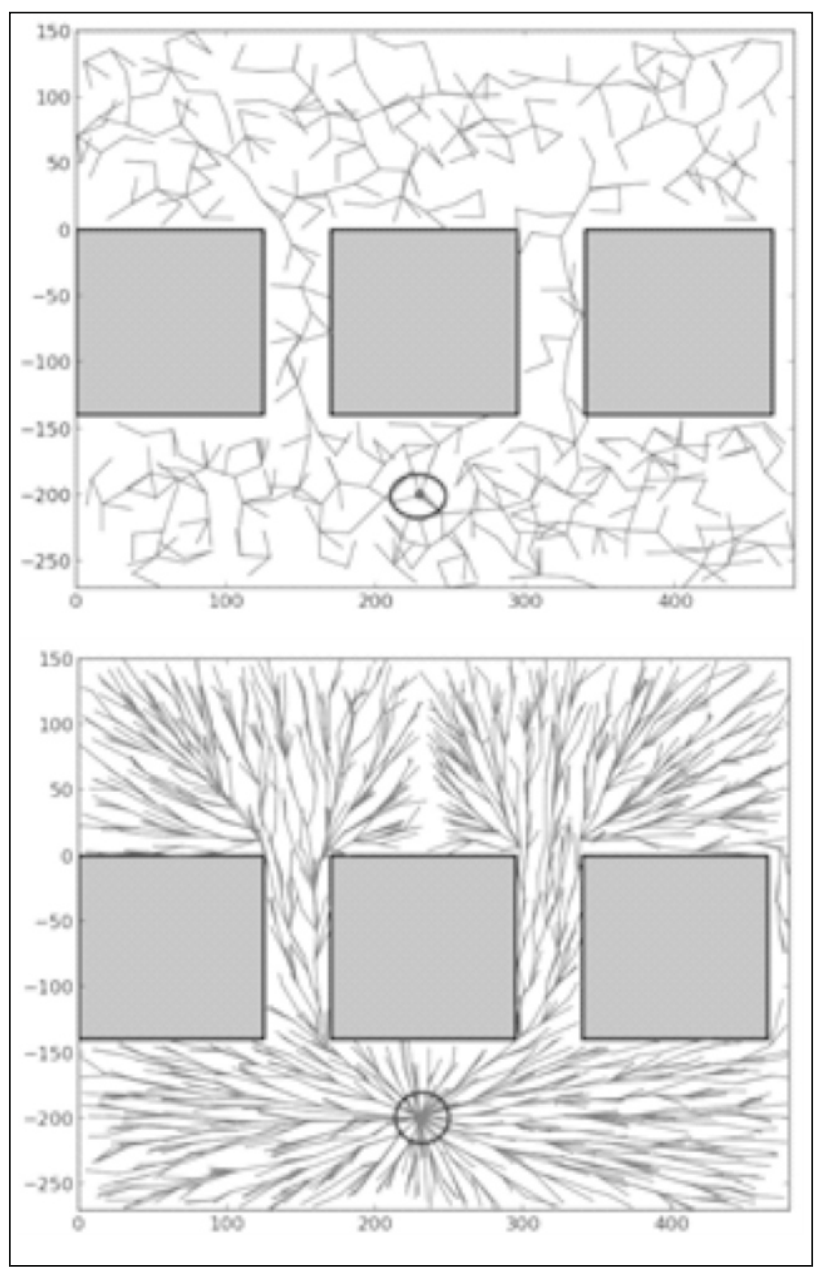

Figura 4. Expansión de un (a) RRT y un (b) RRT* en un entorno 2D. 

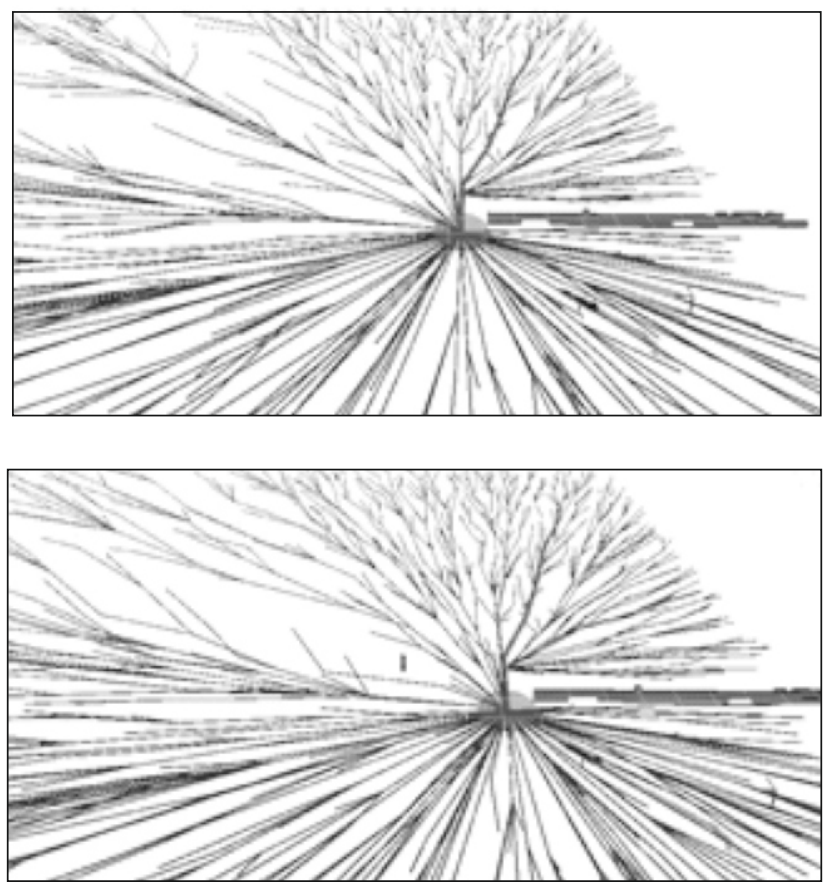

Figura 5. Expansión de un $\mathrm{RRT}^{*}$ en línea. Se aprecia en azul, las diferentes ramas del árbol; la esfera roja corresponde al punto inicial de la consulta y también al punto donde se dirige el vehículo; el camino desde el inicio hasta el final se puede apreciar en rojo, mientras que la trayectoria hecha por el vehículo se observa en

verde. Los vóxel en color morado corresponden a espacios ocupados del entorno representado en un

Octomap. Se observa claramente que una región previamente identificada como libre en (a) se ha marcado como ocupada en (b) generando el descarte de las ramas implicadas en la colisión.

\subsubsection{Coordinador de mission}

El tercer y último bloque funcional del sistema de mapeo y planificación (ver Fig. 2) corresponde al coordinador de misión. Éste funciona como un controlador de alto nivel; asimismo, es el encargado no solo de inicializar los bloques de mapeo y planificación, sino además, de recibir la tarea a realizar, así como comunicar la información de estos bloques funcionales con el controlador del robot.
Adicional a ello, es el encargado de verificar si el camino entre la posición actual del vehículo y los puntos enviados al controlador es seguro; en caso contrario, notificará al controlador del vehículo que se detenga y solicitará al planificador que actualice su mapa y busque un nuevo camino seguro hacia la meta.

\subsection{Función de costo y visibilidad}

Además de la dificultad que implica explorar un entorno submarino sin la posibilidad de disponer de un mapa sobre el cual navegar, obtener información útil para estudiar dicho entorno requiere que las rutas de exploración se definan con base en un criterio que optimice la información obtenida.

Por esta razón, la propuesta de este trabajo es definir un mapa de costos sobre el C-Space, mediante el cual se pueda evaluar la calidad del camino obtenido, en términos de la visibilidad del mismo respecto a la estructura submarina inspeccionada. Este criterio fue propuesto por lo autores previamente con el objetivo de mantener una altitud de un AUV con respecto al fondo marino (Hernández, et al., 2015).

Para la aplicación presentada en este trabajo, se definió un costo q asociado a cada configuración, $0 \leq$ Cost $\leq 100$, donde 0 y 100 son los costos mínimos y máximos, respectivamente, y están dados por la ecuación (1). Como se observa, el costo depende de la distancia (d) e incluye parámetros variables; entre ellos, se encuentra distancia deseada (o esperada) d_e respecto a la estructura inspeccionada.

Otro parámetro variable es el rango de distancia permitido respecto a la distancia deseada, dentro del cual la visibilidad no se ve afectada $\Delta d$ _a. Dicha región, donde la visibilidad se mantiene, equivale a la zona de costo mínimo, como se aprecia en color azul oscuro en la Fig. 6. Por último, es importante anotar que esta función de costo, definida en la Ecuación (1), define entonces el objetivo de optimización para el algoritmo RRT* en línea explicado previamente. 


$$
\operatorname{Cost}(d)=\left\{\begin{array}{cc}
\left(1-\frac{d}{d_{e}}\right) 100, & d<d_{e}-\frac{\Delta d_{a}}{2} \\
0, & d_{e}-\frac{\Delta d_{a}}{2} \leq d \leq d_{e}+\frac{\Delta d_{a}}{2} \\
\left(\frac{d}{d_{e}}-1\right) 100 & d>d_{e}+\frac{\Delta d_{a}}{2}
\end{array}\right.
$$

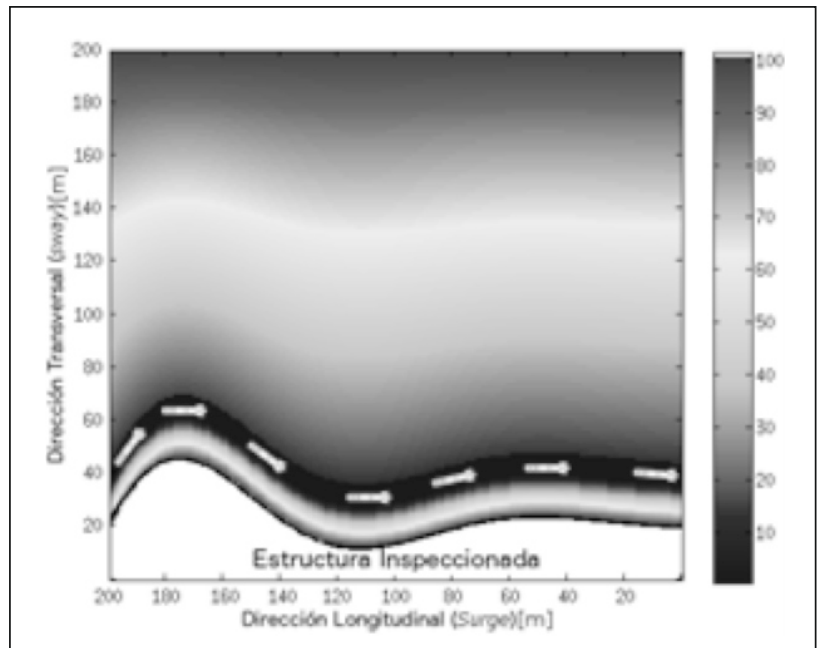

Figura 6. Mapa de costos proyectado en los ejes de movimiento del vehículo en las direcciones longitudinal y transversal. Las regiones de azul oscuro y rojo indican zonas de coste mínimo y máximo, respectivamente.

\section{RESULTADOSY DISCUSIÓN}

Para validar nuestro sistema de mapeo y planificación de caminos para la exploración de entornos submarinos con AUV, se utilizó el SPARUS-II (ver Fig. 1). EI SPARUS-II es un AUV tipo torpedo con capacidad de inmersión máxima de $200 \mathrm{~m}$ de profundidad; este cuenta con tres motores (dos horizontales y uno vertical), que permiten al vehículo, moverse en dirección longitudinal (surge), vertical (heave) y de guiñada (yaw).

El vehículo calcula su posición bajo el agua mediante un sistema de navegación que incluye sensores de presión, de velocidad por efecto Doppler (DVL), sensores inerciales (IMU) y GPS; este último le permite establecer un punto inicial de ubicación cuando está en superficie. Para percibir su entorno, el vehículo cuenta con sensores exteroceptivos acústicos como eocosondas y perfiladores.

Las diferentes pruebas para validar nuestra propuesta se realizaron tanto en simulación como en condiciones reales. El escenario de prueba seleccionado en ambos casos fue una estructura rompeolas que protege el puerto de Sant Feliu de Guíxols, en Girona, España.

Dicha estructura está compuesta por una serie de bloques de hormigón de $14 \mathrm{~m}$ de longitud y $12 \mathrm{~m}$ de ancho, con una separación de 4,5 m de separación entre ellos y una profundidad media de $7 \mathrm{~m}$. Dicho escenario, tanto en el mundo real como en el entorno de simulación, se pueden apreciar en la Fig. 7.

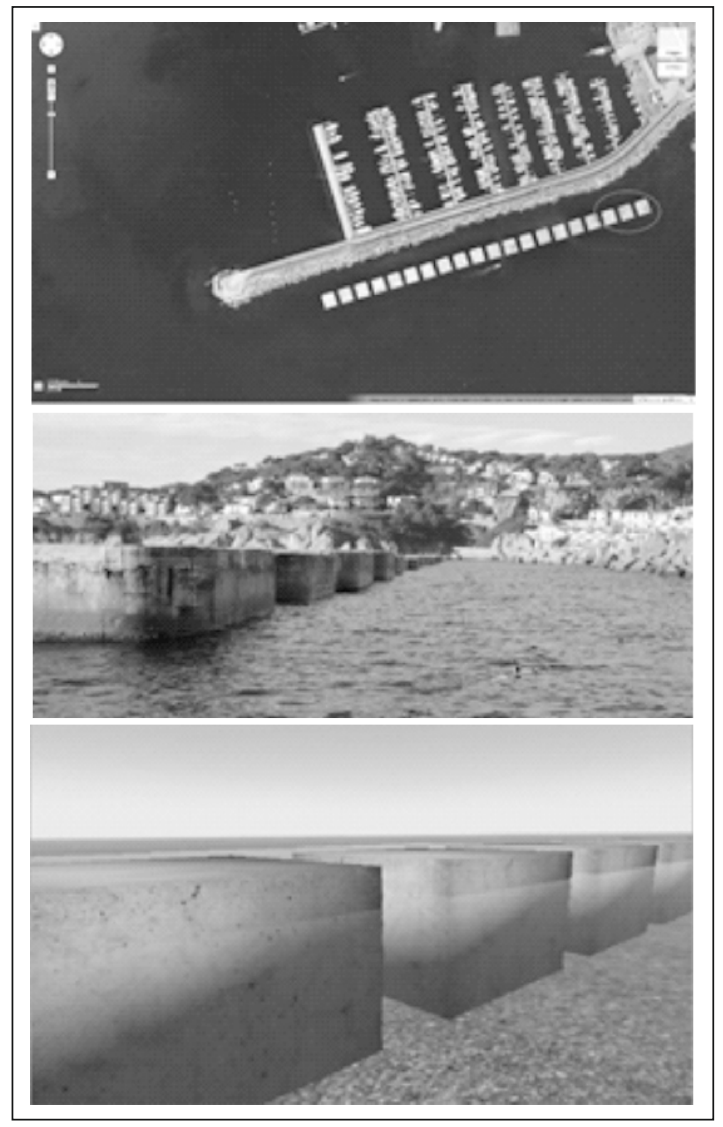

(a)

(b)

Fig. 7. Escenario de prueba y validación. Estructura 
rompeolas compuesta por una serie de bloques de hormigón. (a) Vista aérea. (b) SPARUS-II AUV junto a los bloques de concreto. (c) Escenario de simulación equivalente.

\subsection{Pruebas de simulación: SPARUS-II AUV realizando tareas de inicio-a-meta}

Las diferentes tareas en simulación se definieron de manera que el SPARUS-II tuviera que desplazarse desde un lado del rompeolas al otro, lo que requería navegar en medio de los bloques de hormigón pasando por el espacio de 4,5 m. El entorno se asumía como desconocido y requería mapeo y planificación simultánea y en línea. La Fig. 8 muestra algunas situaciones que explican el proceso completo para completar una tarea inicio-a-meta realizada en el entorno de simulación
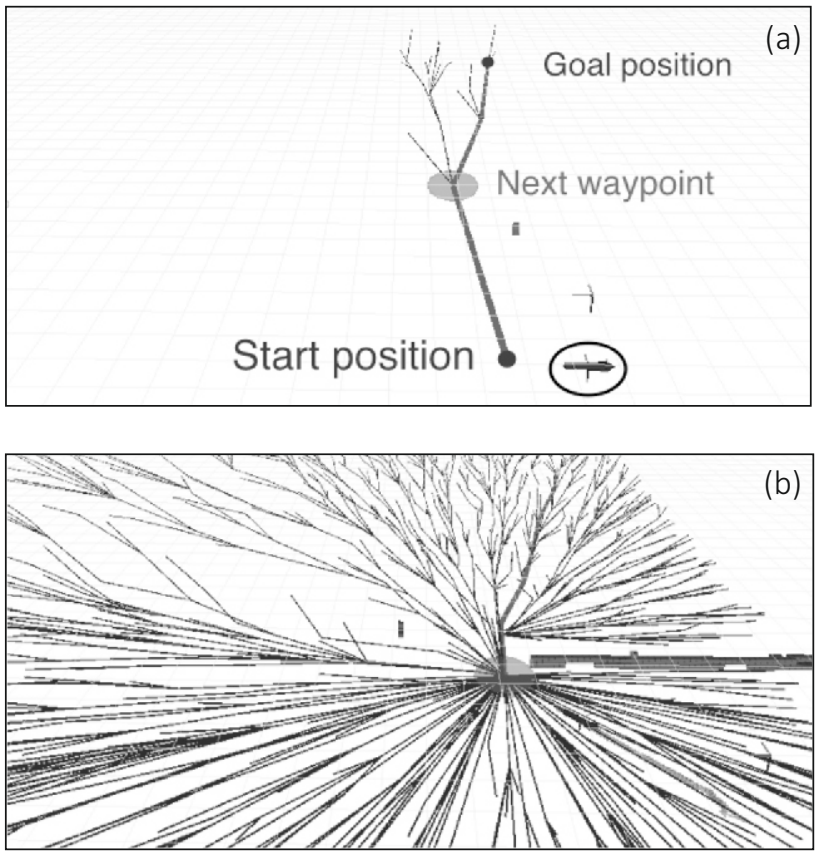
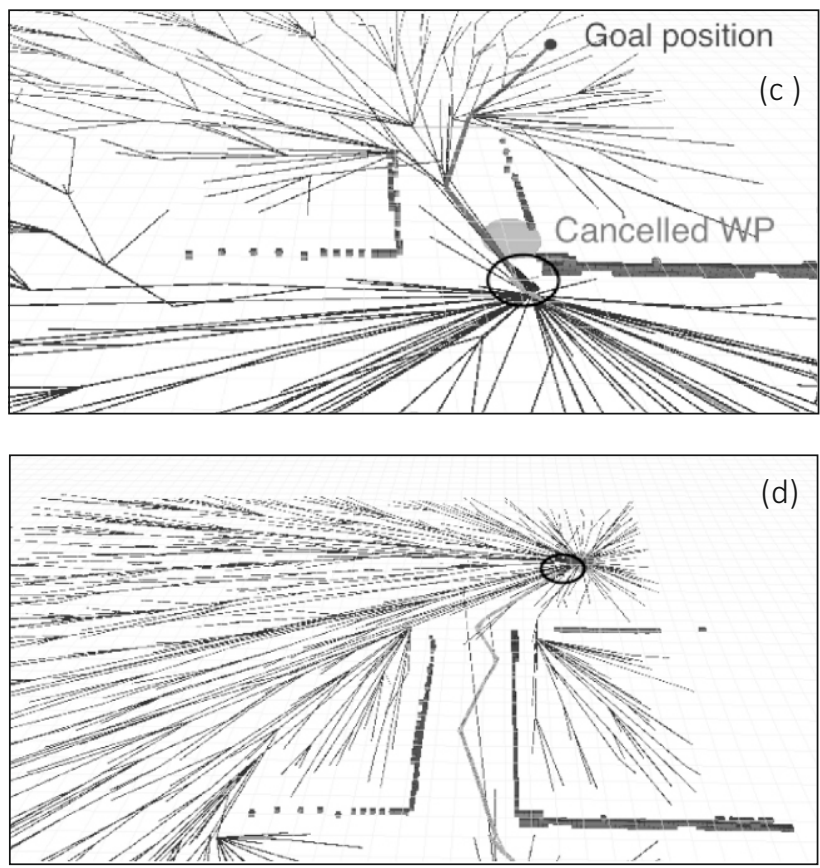

Figura 8. SPARUS-II ejecuta una tarea de inicio-a-meta: (a) el entorno se asume inicialmente como desconocido, (b) lo que requiere mapeo y planificación simultáneo y en línea. (c) Cuando un punto compromete la seguridad del vehículo, el sistema cancela dicho punto y busca un nuevo camino seguro con una versión actualizada del mapa. (d) El AUV completa satisfactoriamente la tarea..

\subsection{Pruebas en el entorno real}

Al igual que en las pruebas en simulación, las pruebas en el entorno real se definieron de manera que el vehículo tuviera que navegar en medio de los bloques de hormigón de la estructura rompeolas. La Fig 9 muestra el resultado de una misión en el mundo real.

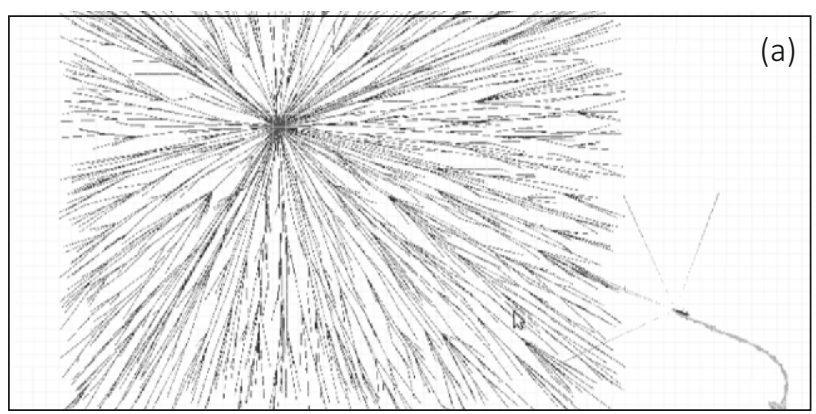




\section{Ingenierías:-.:}
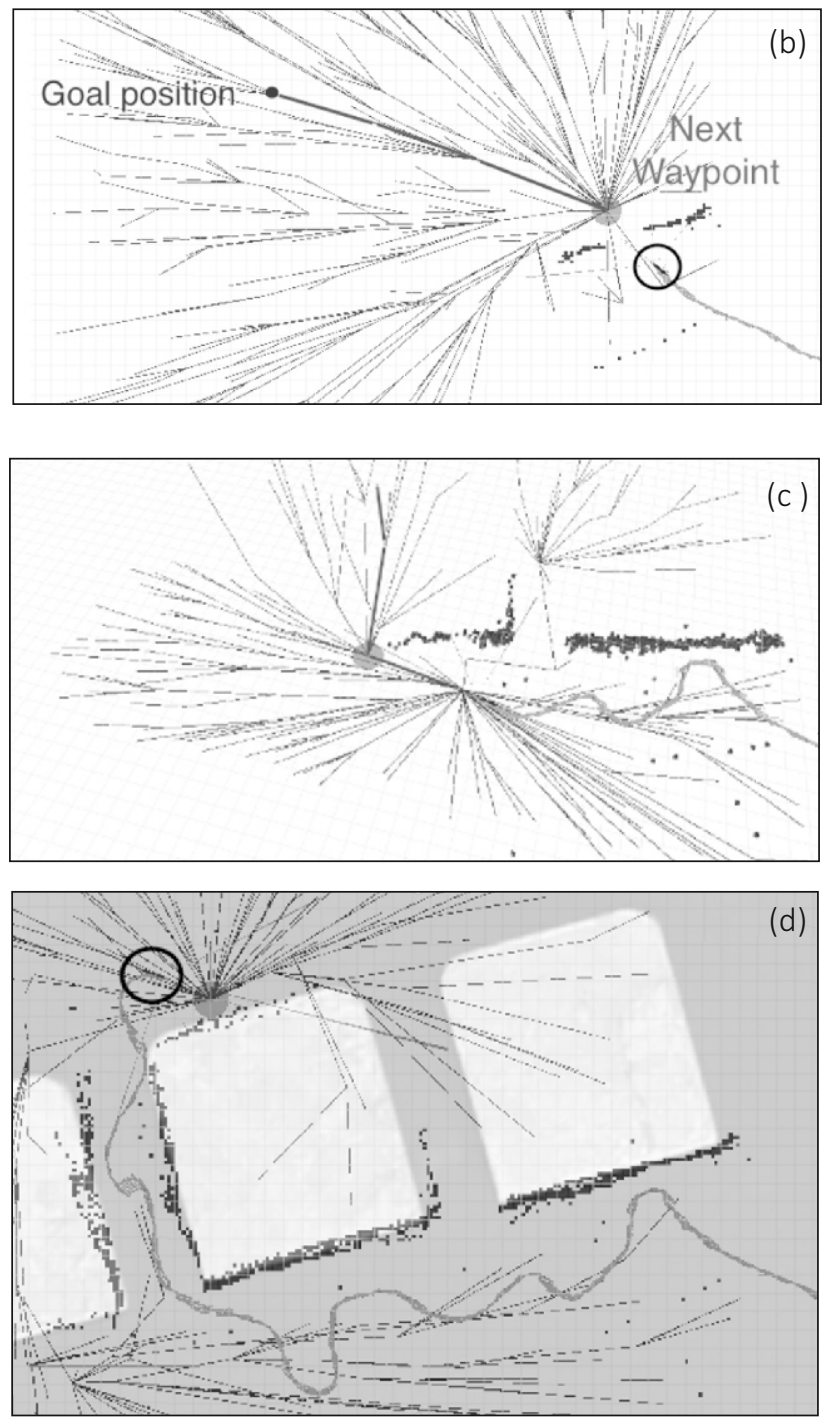

Figura 9. SPARUS-II resuelve una tarea inicio-a-meta que requería ir desde un lado del rompeolas hasta el otro. (a)

Asumiendo el entorno como completamente desconocido, (b) el AUV debe mapear y (re)planificar caminos simultáneamente y en línea. (c) El mapa representa con alta fidelidad los bloques de hormigón. (d) EI AUV completa satisfactoriamente la misión. El mapa resultante se muestra sobre una imagen real satelital del rompeolas.

\section{CONCLUSIONES}

En este artículo se presenta una estrategia que permite a un vehículo autónomo submarino (AUV) navegar en un entorno desconocido. Para ello, se presenta como requisito principal que el vehículo pueda mapear y planificar caminos libres de colisión simultáneamente y en línea. Para lograrlo, el sistema propuesto cuenta con tres bloques funcionales de mapeo, planificación y coordinación, los cuales son explicados en detalle, no solo en su funcionamiento, sino también en la forma como interactúan entre sí.

Para el mapeo incremental y en línea, se propone el uso de Octomaps; estos son estructuras de datos que permiten representar espacios con y sin obstáculos, así como diferenciarlos de áreas no exploradas. Para la planificación de caminos, se presenta una versión modificada del RRT*, un algoritmo basado en el muestreo aleatorio del espacio de configuraciones C-Space. En ambos casos, mapeo y planificación de caminos, diferentes pruebas de simulación y en entornos reales son presentadas que validan la capacidad de navegar en espacios desconocidos de manera segura.

\section{TRABAJO FUTURO}

Durante las diferentes pruebas, de simulación y en entornos reales, se apreció que el sistema debe realizar un alto número de maniobras de replanificación (aquellas donde el vehículo se ve en un situación que el camino no es válido); se ha detectado que en gran medida, éstas se deben a que el planificador no considera las restricciones de movimiento propias del vehículo. Es importante anotar que el SPARUS-II, así como otros vehículos tipo torpedo, son sistemas no-holonómicos. Por ello, una línea de trabajo sería que el planificador genere caminos que cumplan con las restricciones de giro (de movimiento) del vehículo.

Adicionalmente, otra línea de investigación y trabajo futuro a corto plazo es la extensión del sistema de mapeo y planificación para movimientos 3-dimensionales (3D). Sin embargo, es importante destacar que cada uno de los 


\section{. . F.'Ingenierías}

bloques funcionales cuenta con las características necesarias para que esto sea posible, por lo que la extensión depende más de adaptaciones de carácter físico (hardware) del vehículo, que incluyen las modificaciones de sensores que perciben espacios 3D.

\section{AGRADECIMIENTOS}

Este trabajo ha sido financiado por el proyecto morph del séptimo programa marco de la unión europea, acuerdo FP7-ICTE-2011-7-288704. Adicionalmente, J.D. Hernández Vega ha sido financiado parcialmente por el programa de formación de capital humano de alto nivel, a través del programa de formación doctoral del Departamento Administrativo de Ciencia, Tecnología e Innovación (Colciencias).

\section{REFERENCIAS}

Barrientos, A., Peñin, L., Balaguer, C., y Aracil, R. (2007). Fundamentos de Robotica. McGraw-Hill.

Galceran, E., Campos, R., Palomeras, N., Ribas, D., Carreras, M., y Ridao, P. (2014). Coverage Path Planning with Real-time Replanning and Surface Reconstruction for Inspection of Three-dimensional Underwater Structures using Autonomous Underwater Vehicles. Journal of Field Robotics. doi:10.1002/rob.21554

Hernández, J. D., Vallicrosa, G., Vidal, E., Pairet, È., Carreras, M., y Ridao, P. (2015). On-line 3D Path Planning for Close-proximity Surveying with AUVs. En IFAC Workshop on Navigation, Guidance and Control of Underwater Vehicles (NGCUV'2015). Girona.

Hernández, J. D., Vidal, E., Vallicrosa, G., Galceran, E. y Carreras, M. (2015). Online path planning for autonomous underwater vehicles in unknown environments. En IEEE International Conference on Robotics and Automation (ICRA) (pp. 1152-1157). Seattle: IEEE. doi:10.1109/ICRA.2015.7139336

Hornung, A., Wurm, K. M., Bennewitz, M., Stachniss, C. y Burgard, W. (2013). OctoMap: an efficient probabilistic 3D mapping framework based on octrees. Autonomous Robots, 34(3), 189-206. doi:10.1007/s10514-012-9321-0

Hover, F. S., Eustice, R. M., Kim, A., Englot, B., Johannsson, H., Kaess, M. y Leonard, J. J. (2012). Advanced perception, navigation and planning for autonomous in-water ship hull inspection. The International Journal of Robotics Research, 31(12), 1445-1464. doi:10.1177/0278364912461059

Karaman, S. y Frazzoli, E. (2011). Sampling-based Algorithms for Optimal Motion Planning. The International Journal of Robotics Research, 30(7), 846894. doi:10.1177/0278364911406761 


\section{Ingenierías:H : ,}

Kavraki, L. E., Svestka, P., Latombe, J.-C. y Overmars, M. H. (1996). Probabilistic roadmaps for path planning in high-dimensional configuration spaces. IEEE Transactions on Robotics and Automation, 12(4), 566580. doi:10.1109/70.508439

LaValle, S. M. y Kuffner, J. J. (2001). Randomized Kinodynamic Planning. The International Journal of Robotics Research, 20(5), 378-400. doi:10.1177/02783640122067453

Randomized Kinodynamic Planning. The International Journal of Robotics Research, 20(5), 378-400. doi:10.1177/02783640122067453

Maki, T., Mizushima, H., Kondo, H., Ura, T., Sakamaki, T. y Yanagisawa, M. (2007). Real time path-planning of an AUV based on characteristics of passive acoustic landmarks for visual mapping of shallow vent fields. En M T S / I EEE OCEANS. Van couver. doi:10.1109/OCEANS.2007.4449321

Mallios, A., Ridao, P., Carreras, M. y Hernàndez, E. (2011). Navigating and mapping with the SPARUS AUV in a natural and unstructured underwater environment. En MTS/IEEE OCEANS. Waikoloa. Recuperado a partir de:

http://ieeexplore.ieee.org/xpls/abs_all.jsp?arnumbe $r=6107105$

Petillot, Y., Ruiz, I. T., y Lane, D. M. (2001). Underwater vehicle obstacle avoidance and path planning using a multi-beam forward looking sonar. IEEE Journal of Oceanic Engineering, 26(2), 240-251. doi:10.1109/48.922790. 